Archives de sciences sociales des religions

172 | octobre-décembre

Bulletin Bibliographique

\title{
Émile Poulat et la crise de la théologie en 1950
}

Denis Pelletier

\section{OpenEdition}

Journals

Édition électronique

URL : http://journals.openedition.org/assr/27257

DOI : $10.4000 /$ assr.27257

ISSN : $1777-5825$

Éditeur

Éditions de l'EHESS

Édition imprimée

Date de publication : 1 octobre 2015

Pagination : 201-207

ISBN : 978-2-7132-2515-4

ISSN : 0335-5985

Référence électronique

Denis Pelletier, "Émile Poulat et la crise de la théologie en 1950 », Archives de sciences sociales des religions [En ligne], 172 | octobre-décembre, mis en ligne le 26 avril 2018, consulté le 03 mai 2019. URL : http://journals.openedition.org/assr/27257 ; DOI : 10.4000/assr.27257 


\title{
Denis Pelletier
}

\section{Émile Poulat et la crise de la théologie en 1950}

\author{
À propos de : \\ POULAT Émile, Le désir de voir Dien et sa signification pour la théologie \\ française contemporaine, \\ suivi d'un entretien avec Yvon Tranvouez et François Trémolières. \\ Introduction et édition du texte par François Trémolières, biblio- \\ graphie par Yvon Tranvouez, Paris, Desclée de Brouwer, 2015, \\ $360 \mathrm{p}$.
}

Le 9 janvier 1950, Émile Poulat, qui achevait une année de lectorat à l'université de Fribourg-en-Brisgau en Allemagne, y soutint une thèse de doctorat de théologie rédigée en français et intitulée "L'interprétation du désir naturel de voir Dieu et sa signification dans la théologie française contemporaine ». Restée longtemps ignorée, cette thèse, écrite avant son rattachement à la Mission de Paris, paraît quelques mois après sa mort en novembre 2014, dans une édition augmentée d'un chapitre supplémentaire, intitulé "Antagonismes et affinités » et consacré aux débats suscités par le philosophe Maurice Blondel, chapitre qu'il rédigea en 1950 en vue d'une publication qui ne vint pas. Elle est accompagnée de la transcription d'un entretien avec les historiens Yvon Tranvouez et François Trémolières, réalisé peu avant sa mort, ainsi que d'un court texte intitulé «Testament spirituel » et daté de 2011. Responsable de la publication, François Trémolières propose également une longue introduction critique à la thèse, qui est suivie d'une bibliographie complète d'Émile Poulat réalisée par Yvon Tranvouez ${ }^{1}$. L'ensemble constitue un travail éditorial d'une qualité exceptionnelle, un livre dont la lecture est, de bout en bout, passionnante. Il s'inscrit notamment dans un ensemble de travaux, récents ou en cours, qui revisitent l'histoire des sciences sociales du religieux dans leur rapport au champ théologique. Mais il ne s'y réduit pas : rédigée au moment de la polémique sur la «nouvelle théologie » entre les dominicains de La revue thomiste et les jésuites de l'« École de Fourvière », la thèse est une pièce supplémentaire à l'histoire de

1. À partir de celle publiée dans l'ouvrage collectif dirigé par Valentine Zuber et Jean Baubérot, Un objet de science, le catholicisme. Réflexions autour de l'ouvre d'Émile Poulat, Paris, Bayard, 2001, p. 291-326. 
la théologie française au $\mathrm{XX}^{\mathrm{e}}$ siècle, quel qu'ait été l'itinéraire ultérieur de son auteur.

Partons donc de la thèse, non pour la résumer mais pour tenter d'en ressaisir le mouvement. "Le désir de voir Dieu : tel est l'un des thèmes permanents de la pensée chrétienne ", écrit Poulat en tête du premier chapitre (p. 67). Mais en faisant de ce désir un désir naturel ( «desiderium naturale»), Thomas d'Aquin a ouvert un espace de controverse qui ne s'est jamais refermé, et qui s'inscrit dans la longue durée du débat sur le rapport entre nature et surnaturel. Quel lien existe-t-il en effet entre un désir naturel et sa fin surnaturelle, qui est ici la vision béatifique, dès lors qu'en bonne logique aristotélicienne, il n'est point de désir qui ne puisse aboutir à sa fin selon son ordre propre ? Et qu'en est-il de la grâce, et de sa gratuité, si elle est en quelque manière tenue, voire obligée, par l'existence de ce désir naturel ?

De cette controverse, les cinq premiers chapitres construisent une généalogie à la fois érudite, attentive à la lettre des textes, et pourtant déjà discrètement orientée. En aval au plan chronologique, mais en amont de l'enquête menée par Poulat, il y a les polémiques contemporaines. Dans son premier chapitre, il développe la question telle qu'elle traverse la théologie française depuis la thèse de philosophie de Maurice Blondel (1893), qui en a changé la donne en posant "l'action » comme fondement de la connaissance dépassant l'écart entre expérience et effort spéculatif, entre immanence et transcendance, entre nature et surnaturel enfin. D’un côté, de Pierre Rousselot à Henri de Lubac en passant par Guy de Broglie et Yves de Montcheuil, une lignée de réception majoritairement jésuite prend en compte le tournant opéré par Blondel tout en l'inscrivant dans un retour à Thomas d'Aquin contre un néothomisme qui le prive de son histoire et réduit la théologie à une science des conclusions exclusivement préoccupée d'orthodoxie logique. De l'autre, Réginald Garrigou-Lagrange, Pedro Descoqs et leurs alliés, condamnent l'anti-intellectualisme des premiers et leur reprochent de céder à l'immanentisme. L'ensemble débouche sur l'affaire de la " nouvelle théologie ", qui oppose depuis 1946 les dominicains de la Revue thomiste aux jésuites de Fourvière ${ }^{2}$, et que Poulat évoque brièvement et sans pathos (p. 78-79).

En amont, il y a Thomas d'Aquin. Après avoir longuement cité les passages où se noue la question du desiderium naturale (chapitre 2), Émile Poulat livre par approches successives - la transcendance au chapitre 3, le désir et sa fin au chapitre 4, la finalité de l'esprit au chapitre 5 - le portrait d'un philosophe au travail avec la pensée antique et ses inflexions chrétiennes. « Le premier, il tenta

2. Pièces du dossier dans la brochure de M. Labourdette, M.-J. Nicolas, R.-L. Bruckberger, o.p., Dialogue théologique, Pièces du débat entre "La Revue Thomiste » et les RRPP de Lubac, Bouillard, Fessard, von Balthasar, S. J., d'autre part, Saint-Maximin, 1947. Voir surtout Étienne Fouilloux, Une Église en quête de liberté, La pensée catholique française entre modernisme et Vatican II 1914-1962, Paris, Desclée de Brouwer, 1998, p. 193-244. 
la synthèse de deux conceptions de la vie, l'une d'origine biblique, l'autre d'origine grecque » (p. 187). Thomas invente une philosophie de la nature qui dégage Aristote de son naturalisme, il cherche un équilibre subtil et finalement inachevé entre « la philosophie platonicienne de la participation » à la divinité, reprise par la tradition néoplatonicienne des pères de l’Église, et la " philosophie aristotélicienne de l'expérience et du réel ». La question du désir naturel de voir Dieu maintient la raison philosophique ouverte à l'enjeu de la foi et à l'aspiration mystique, au rebours de la prétention d'une partie du thomisme contemporain à réserver à la théologie l'accès légitime à la transcendance. Pour autant, ce désir « ne menace ni la transcendance ni la gratuité du surnaturel» (p.150): son ressort n'est pas dans la psychologie mais dans cette " loi intime de notre intelligence » (p. 140) qu'est le désir de connaître les causes, une "capacité passive » (p. 149) qui nous conduit de la connaissance de l'existence de Dieu vers le désir d'en connaître l'essence, auquel répond la vision béatifique. Ainsi Thomas estil demeuré plus "augustinien » que ne l'ont pensé les modernes : chez lui, la notion de nature ne se construit pas en opposition au surnaturel, mais dans une relation dialectique avec la gratuité qui caractérise l'intervention de Dieu. L'ordre de la nature n'est pas un ordre clos sur lui-même - ce sera là une invention ultérieure, et une forme d'oubli de son œuvre.

Cet oubli prend dans la thèse la forme d'un récit de controverse, quasi entièrement inscrit dans la théologie spéculative, où le détour par la méthode historienne vient parfois poser des jalons pour la suite. Le récit connaît des méandres techniques - puissance obédientielle, désir élicite, degrés de la transcendance... -, des acteurs principaux et secondaires. Poulat cite beaucoup, il est attentif à la lettre des textes et à leur positionnement au regard de l'institution, toutes choses au travers desquelles le jeune théologien montre son érudition et donne les gages d'une orthodoxie compétente. La controverse sur la nature connaît un tournant à l'époque moderne : avec Thomas Cajetan (1469-1534), la théorie de la « nature pure " "fait une entrée discrète dans la théologie, corrélativement à la "nature historique" ; avec Billuart, ce qui était simple hypothèse est devenu l'état normal de l'être créé en puissance obédientielle à l'égard de la grâce» (p. 165). Le Summa Sancti Thomae de Charles René Billuart (1685-1757) est paru entre 1746 et 1751 . Autrement dit, de la polémique antiprotestante au combat contre le baïanisme puis le jansénisme, la théorie de la pure nature a ouvert la voie à une opposition entre nature et surnaturel qui est à l'opposé du vrai Thomas d'Aquin.

Émile Poulat consacre les deux derniers chapitres de son livre à une récapitulation critique de l'ensemble du débat, dont le pivot est la thèse de Blondel sur L'action (1893). Parce qu'elle ouvrait une indécision sur la relation entre un désir naturel et sa fin surnaturelle, la controverse sur le « désir naturel de voir Dieu » met en marche une série de couples dont les termes ont fini par s'exclure aux yeux de certains : nature/surnaturel, philosophie/théologie, immanence/ transcendance, etc. Chaque camp a ses forces et ses faiblesses, mais la cible 
principale est clairement la scolastique tardive, celle qui court de Cajetan et Suarez à Garrigou-Lagrange et Boyer, et qu'Henri de Lubac qualifie non sans malice de «moderne ". À la théologie mystique de Thomas d'Aquin, elle a substitué une discipline purement spéculative, qui s'est coupée de l'esprit de son temps pour devenir une "science des conclusions ", intolérante et fermée à ses interlocuteurs. En inventant chez Thomas d'Aquin une opposition entre nature et surnaturel qui n'y était pas, elle est devenue complice du naturalisme, cette conception profane de l'ordre du monde qui a fait le lit de la séparation entre le christianisme et la pensée moderne. "Sous le signe de la séparation ", tel est le titre du chapitre 6 : la France du Xx siècle, dont le débat intellectuel est structuré par la laïcité, est un observatoire privilégié de ce mécanisme par lequel « le régime de la séparation fut celui de la philosophie et de la théologie, avant de devenir le fait de l’Église et de l'État» (p. 54).

À lire cette phrase écrite en 1949, on reste songeur sur la capacité de l'historiographie à renouveler la problématique depuis plus de soixante ans. Mais on comprend le rôle tenu par Blondel dans la thèse de Poulat. Comment sauver la présence du catholicisme au sein d'une société qui se déprend de la transcendance, avec la complicité active et paradoxale de théologiens qui croient faire œuvre apologétique en découvrant chez Thomas d'Aquin une série de lignes de séparation qui n'y figurent pas ? Contre l'usage abusif de l'inquiétude pascalienne à des fins apologétiques, contre l'utopie de son maître Léon Ollé-Laprune rêvant de dépasser Kant avec les outils de Kant, Blondel a posé comme incontournable le principe d'immanence, et c'est sur lui qu'il construit une « dialectique purement métaphysique» (p. 220) entre action et connaissance - on dirait aujourd'hui une théorie de la connaissance fondée dans une phénoménologie de l'action. Sous un titre assez limpide, "l'appel du transcendant dans le déploiement de l'agir» (p. 224), Poulat décrit les étapes par lesquelles passe Blondel pour formuler cette théorie de la connaissance qui résiste activement au «morcelage ontologique» (p. 227). En s'appuyant sur plusieurs de ceux qui l'ont commenté, Pierre Rousselot, Auguste Valensin, Yves de Montcheuil, Étienne Gilson, Jacques Maritain, il montre comment Blondel découvre la nécessité de la transcendance dans le mouvement même de la connaissance qui procède de l'action. «L'action, dont le déploiement laisse disponible en nous un surplus de réalité spirituelle, sait qu'elle ne peut s'achever dans l'ordre naturel » (p. 229), en sorte que c'est comme en creux, selon un via negationis qui fait discrètement écho à l'expérience mystique, que Blondel est conduit par une pensée de l'immanence à faire l'épreuve de l'impossible frontière entre nature et surnaturel. " Le surnaturel n'est donc pas une question dernière qu'on peut se poser comme si la réponse devait laisser toutes choses en leur état premier»(p. 247), mais bien une exigence du sens, découverte a posteriori certes, mais comme fondatrice de la possibilité même de penser l'expérience du monde dans son unité. L'objet de la thèse de Poulat, un désir naturel ordonné à une fin surnaturelle, trouve son 
aboutissement dans cette "assomption théologique de la nature " (p. 243) par quoi se clôt son dernier chapitre.

Bien sûr, il est impossible de lire cette thèse sans s'interroger sur l'itinéraire ultérieur de son auteur. Comment la thèse de théologie de Poulat et son œuvre d'historien et de sociologue s'éclairent-elles réciproquement, quelles relations de continuité ou de rupture entretiennent-elles l'une avec l'autre ? Questions un peu vaines, sans doute, où affleure l'illusion de rendre son unité à une vie d'auteur, et dont sociologues et philosophes nous ont appris à nous méfier voici bien des années déjà. Mais questions dont on ne peut faire l'économie, dans cette revue particulièrement, pour cette simple raison qu'Émile Poulat a fortement contribué par son œuvre à construire le champ des sciences sociales du religieux en France.

On est particulièrement reconnaissant à François Trémolières d'avoir su éviter deux écueils. Le premier aurait consisté à déceler dans la thèse de théologie les traces annonciatrices de ce qui ferait ultérieurement le meilleur de l'œuvre de l'historien et sa part originale. Le second aurait été de s'interroger sans fin sur la continuité et la rupture entre un "premier » et un "second » Poulat. L'intéressé plaidait pourtant lui-même en 2014 pour le premier terme de l'alternative : « J'assume tout ce que j'ai fait, dans sa continuité. Je n'ai jamais eu le sentiment d'une rupture dans mon régime intellectuel » (p. 296). Il n'existe pas de raison valable de mettre en doute ce jugement sur soi d'un homme au soir de sa vie, mais François Trémolières rappelle aussi le silence par lequel Émile Poulat a longtemps conservé dans l'ombre cet aspect de son œuvre, et la grande réserve dont il a fait preuve en levant très tardivement le voile. À s'en tenir à cette approche strictement biographique, Trémolières cite aussi la manière dont Poulat évoquait en 2008 la possibilité d'une "nuit critique » analogue à celle des mystiques, sans qu'il soit possible de dire ni ce que cette nuit aurait mis en crise ni ce qui aurait peut-être pu en marquer le terme.

On peut aussi repérer dans la thèse ce qui fait écho rétrospectif à l'œuvre ultérieure de Poulat, par exemple la double chronologie intellectuelle dans laquelle il n'a cessé de se mouvoir ensuite, chronologie courte qui conduit de la crise du modernisme chrétien au temps présent, chronologie longue d'une déprise moderne du religieux qui s'amorce avec la Renaissance et constitue l'univers de référence du catholicisme intransigeant à l'époque contemporaine. Des thématiques, aussi : la crise du modernisme, encore, qui l'occupera durant de longues années, depuis sa thèse de $1962^{3}$ jusqu'au volume Modernistica paru vingt ans plus tard ${ }^{4}$ - et le réseau de la Sapinière, cette internationale intégriste clandestine construite sous Pie X par Mgr Benigni dont il éditera le dossier en $1969^{5}$, et

\footnotetext{
3. Histoire, dogme et critique dans la crise moderniste, Tournai, Casterman, 1962.

4. Modernistica. Horizons, physionomies, débats, Paris, Nouvelles Éditions latines, 1982.

5. Intégrisme et catholicisme intégral. Un réseau secret international antimoderniste : la "Sapinière ", Tournai, Casterman, 1979.
} 
dont la mise en perspective nourrira ensuite son modèle du catholicisme intransigeant ${ }^{6}$, est lui-même brièvement évoqué dans le chapitre supplémentaire à la thèse (p. 265) ; la séparation, dans sa double dimension intellectuelle et politique, et c'est autour de la seconde qu'il reprit ses recherches au cours des deux dernières décennies de sa vie, renouant avec la pratique de l'édition critique de sources $^{7}$; la mystique enfin, centrale du fait même de l'objet de la thèse et dont il réunira une partie des travaux qu'il lui a consacrés dans deux livres, Critique et mystique (Centurion, 1984) et L'université devant la mystique (Salvator, 1999). De la thèse se dégage enfin une méthode, celle d'un historien des controverses intellectuelles, qui en mesure aussi le contenu politique, pour autant qu'il y ait bien une politique de l'Église dont le débat théologique est un des champs de déploiement. Poulat est, on l'a déjà souligné, particulièrement attentif à la lettre des textes, moins sans doute par usage philologique que du fait d'un habitus théologien formé à l'usage des " autorités ", qu'il utilise non comme telles mais parce que leur mise en réseau balise et oriente l'espace de la controverse. C'est, au fond, d'une histoire des concepts qu'il s'agit, de leur contenu et de leur puissance de représentation et de mobilisation, et Poulat use là d'une forme particulière de la critique, qui s'efface derrière les auteurs convoqués et les laisse organiser peu à peu le paysage qu'il décrit.

Était-ce prudence vis-à-vis de l'institution ? Au fond, la tentative de construction d'un système de correspondances entre la thèse et l'œuvre ultérieure nous renvoie au moment où la première fut écrite. François Trémolières rappelle à juste titre combien les questions abordées par Poulat étaient sensibles à la fin des années 1940, depuis les remous qu'avait suscités la publication en 1946 du livre d'Henri de Lubac, Surnaturel. Études historiques, que suivirent les polémiques sur la "nouvelle théologie ». Il faut donc se représenter Émile Poulat, jeune théologien à peine trentenaire soutenant à Fribourg une thèse de théologie dont les implications sont d'une actualité vive. Son camp est indiscutablement celui de "l'aile marchante» de la théologie française dont Étienne Fouilloux s'est fait l'historien dans Une Église en quête de liberté. Ses références, outre Blondel, vont en priorité à Henri de Lubac et à la lignée dont se réclament les fondateurs de la collection "Sources chrétiennes ${ }^{8}$ ", et il s'aide à plusieurs

6. Voir les deux volumes complémentaires publiés chez Casterman en 1977, Catholicisme, démocratie et socialisme. Le mouvement catholique et Mgr Benigni de la naissance du socialisme à la victoire du fascisme, et Église contre bourgeoisie. Introduction au devenir du catholicisme actuel.

7. Voir notamment Notre laïcité publique, Paris, Berg International, 2003, et l'édition, à la demande en 2003 du premier ministre Jean-Pierre Raffarin et dans le cadre de la commémoration du centenaire de la Loi de 1905, du dossier des associations cultuelles diocésaines, Les Diocésaines. République française, Église catholique : loi de 1905 et associations cultuelles, le dossier d'un litige et de sa solution (1903-2003), Paris, La Documentation française, 2007.

8. Étienne Fouilloux, La collection "Sources chrétiennes ». Éditer les Pères de l'Église au $\mathrm{XX}^{\mathrm{e}}$ siècle, Paris, Le Cerf, 1995. 
reprises de la lecture des Pères de l'Église pour rendre à la théologie sa tradition « mystique » et redécouvrir par là l'originalité oubliée de Thomas d'Aquin. Elles vont aussi à d'autres acteurs du "retour à Thomas ", un Gilson du côté de l'histoire ou un Maritain. Tout se passe comme s'il circulait entre la fraction la plus ouverte du thomisme français du Xxe siècle et les héritiers de Blondel, non sans discuter les uns et les autres, non sans porter aussi une attention réelle à leurs adversaires, en quête de la place que la controverse peut assigner au catholicisme au sein d'une modernité qui s'en écarte. L'objet qu'il s'est choisi peut apparaître désuet, d'une technicité qui décourage l'analyse et semble en garantir l'innocuité. Il pourrait même n'être qu'un prétexte, comme Poulat le laisse luimême entendre dans sa conclusion (p. 251) ; il est plutôt un poste d'observation, presque un laboratoire, solidement construit à partir du "pas de côté » qui permet à la critique de se déployer.

Car il y a un élément discrètement corrosif dans le texte, qui est la manière dont un enjeu de théologie se résout dans la philosophie - celle de Blondel -, au prix d'un travail de critique historique, sans que puisse être contestée sur le moment la validité théologique de la démarche : lors de sa soutenance, Poulat obtient la mention "magna cum eruditione ". "Que ma thèse ait pu être acceptée comme thèse de théologie, confie-t-il à ses interlocuteurs de 2014, c'est peutêtre un signe de la crise que la théologie traversait déjà » (p. 296). Je ne crois pas céder à l'illusion rétrospective en soulignant combien la notion d'une théologie en crise travaille à l'intérieur même de la thèse, dans la manière dont la philosophie ne cesse d'en bousculer la légitimité à dire le vrai, et l'emporte finalement sur elle à travers Blondel - à moins qu'elle ne lui sauve la mise, ce qui ne vaut guère mieux. C'est là un des biais par lesquels la comparaison avec Michel de Certeau, suggérée à Poulat par Trémolières, mériterait sans doute d'être creusée. Dès lors, il importe peu que Le désir de voir Dieu ait été une "grande » ou une « petite » thèse - c'est un débat qu'il faut laisser à d'autres lieux que les Archives. Poulat n'a pas la verve polémique de Jean Daniélou, lui est étrangère la colère blanche qui sourd de certaines pages d'Henri de Lubac, dont le sépare en outre son refus de l'apologétique. Mais son travail est porté par une critique d'autant plus efficace qu'elle se déploie peu à peu, sur un mode détaché, presque subreptice, dans l'entrelacs des références et des concepts. Nous sommes en 1950, un jeune théologien est au travail, il éprouve ses outils, mesure leur capacité de résistance, sélectionne ceux qui lui paraissent forgés pour le combat, fussent-ils indésirables à d'autres. Rien, dans sa thèse, n'autorise à penser qu'il deviendra historien.

Denis PELLETIER

GSRL - UMR 8582 EPHE-CNRS denis.pelletier@ephe.sorbonne.fr 
